04

\title{
Исследование наносекундного разряда в аргоне при атмосферном давлении с предварительной ионизацией
}

\author{
(C) В.С. Курбанисмаилов ${ }^{1}$, О.А. Омаров ${ }^{1}$, Г.Б. Рагимханов ${ }^{1, \uparrow}$, Д.В. Терешонок ${ }^{2}$ \\ ${ }^{1}$ Дагестанский государственный университет, Махачкала, Россия \\ ${ }^{2}$ Объединенный институт высоких температур РАН, Москва, Россия \\ ฯ E-mail: gb-r@mail.ru
}

Поступило в Редакцию 17 апреля 2018 г.

С применением высокоскоростного фотоэлектронного регистратора с наносекундным временны́м разрешением и на основе двумерной осесимметричной диффузионно-дрейфовой модели выполнено исследование влияния начальных условий на особенности формирования и развития катодонаправленной волны ионизации между двумя плоскими электродами в аргоне при атмосферном давлении.

DOI: 10.21883/PJTF.2019.02.47213.17334

Несмотря на большое число публикаций, посвященных исследованию импульсных объемных разрядов (OP), многие вопросы, связанные с физикой формирования начальных стадий, вызывают научные дискуссии [1-5].

В настоящей работе в условиях равномерной предварительной ионизации газа аргона при атмосферном давлении выполнено как экспериментальное, так и расчетно-теоретическое исследование формирования импульсного ОР.

Подробное описание экспериментальной установки, а также методики исследования представлено в работе [5]. Амплитуда напряжения на разрядном промежутке изменялась в диапазоне $U_{0}=3-20 \mathrm{kV}$. Начальная ионизация газа была на уровне $n_{0} \sim 10^{8} \mathrm{~cm}^{-3}$ и создавалась посредством облучения через сетчатый анод диаметром $4 \mathrm{~cm}$ ультрафиолетом от стороннего искрового разряда. В качестве катода использовался цельный диск диаметром $4 \mathrm{~cm}$ из нержавеющей стали. Расстояние между параллельными электродами составляло $d=1 \mathrm{~cm}$. Динамика формирования разряда с пространственным и временны́м разрешением в наносекундном диапазоне времени исследовалась с применением фотоэлектронного регистратора (ФЭР2-1).

На рис. 1 приведены пространственные картины формирования ОР в аргоне. При создании начальной концентрации электронов в промежутке $n_{0} \approx 10^{8} \mathrm{~cm}^{-3}$ и незначительных перенапряжениях $W=10-100 \%$ первое регистрируемое свечение возникает на аноде к началу резкого роста тока и распространяется к катоду со скоростью $\sim(2-5) \cdot 10^{7} \mathrm{~cm} / \mathrm{s}$. На стадии формирования и распространения фронта свечения ток разряда меняется в пределах 1-10 А.

По мере продвижения фронта свечения к катоду концентрация электронов в разрядном промежутке увеличивается. Величина концентрации электронов, оцененная по сечению разряда и плотности тока, в уже сформировавшемся плазменном столбе составляет $\sim 10^{13}-10^{14} \mathrm{~cm}^{-3}$. Перекрытие ионизационным фронтом разрядного промежутка приводит к образованию катодного пятна (рис. 1, кадр 4), из которого прорастает высокопроводящий искровой канал со скоростью $V_{c} \approx 2 \cdot 10^{6} \mathrm{~cm} / \mathrm{s}$.

Моделирование выполнено в двумерной осесимметричной постановке. Расчет проводится в аргоновой среде при атмосферном давлении в условиях, аналогичных эксперименту. Напряжение на электродах задавалось исходя из эксперимента для случая, когда амплитуда напряжения на разряднике составляла $6.8 \mathrm{kV}$.

Расчетная сетка по радиусу была равномерной с числом ячеек $N_{r}=25$. В межэлектродном промежутке сетка сгущалась вблизи электродов $N_{z}=250$. При этом увеличение числа ячеек по радиусу до 200 (по оси $z$ количество ячеек не меняется) не приводит к существенному отличию решения.

Газоразрядная плазма рассматривается как сплошная многокомпонентная среда, состоящая из нейтральных атомов $(\mathrm{Ar})$, электронов $(e)$, возбужденных атомов $\left(\mathrm{Ar}^{*}\right)$ с энергией возбуждения $11.5 \mathrm{eV}$, атомарных $\left(\mathrm{Ar}^{+}\right)$и молекулярных $\left(\mathrm{Ar}_{2}^{+}\right)$ионов. Кинетика рассматриваемых процессов, константы соответствующих реакций и потери энергии электрона были взяты из работы [6].

Приведенная далее система уравнений (1) включает в себя уравнения баланса заряженных и возбужденных частиц, уравнение для энергии электронов и уравнение Пуассона [7-10]. Нагрев нейтрального газа не учитывался. Температура тяжелых частиц в процессе расчета предполагалась равной температуре нейтрального газа $(300 \mathrm{~K})$.

$$
\begin{gathered}
\frac{\partial n}{\partial t}+\nabla \cdot \boldsymbol{\Gamma}=S \\
\boldsymbol{\Gamma}=q n \mu \mathbf{E}-\nabla(D n), \\
\frac{\partial}{\partial t}\left(\frac{3}{2} n_{e} k_{\mathrm{B}} T_{e}\right)+\nabla \cdot \mathbf{F}=Q_{E}-Q_{e l}-Q_{i n}, \\
\mathbf{F}=\frac{5}{2} k_{\mathrm{B}} T_{e} \boldsymbol{\Gamma}_{e}-\nabla\left(\lambda_{e} T_{e}\right) \\
\lambda_{e}=\frac{5}{2} n_{e} D_{e} \\
\nabla \cdot \mathbf{E}=\frac{e\left(n_{\mathrm{Ar}^{+}}+n_{\mathrm{Ar}_{2}^{+}}-n_{e}\right)}{\varepsilon_{0}}
\end{gathered}
$$



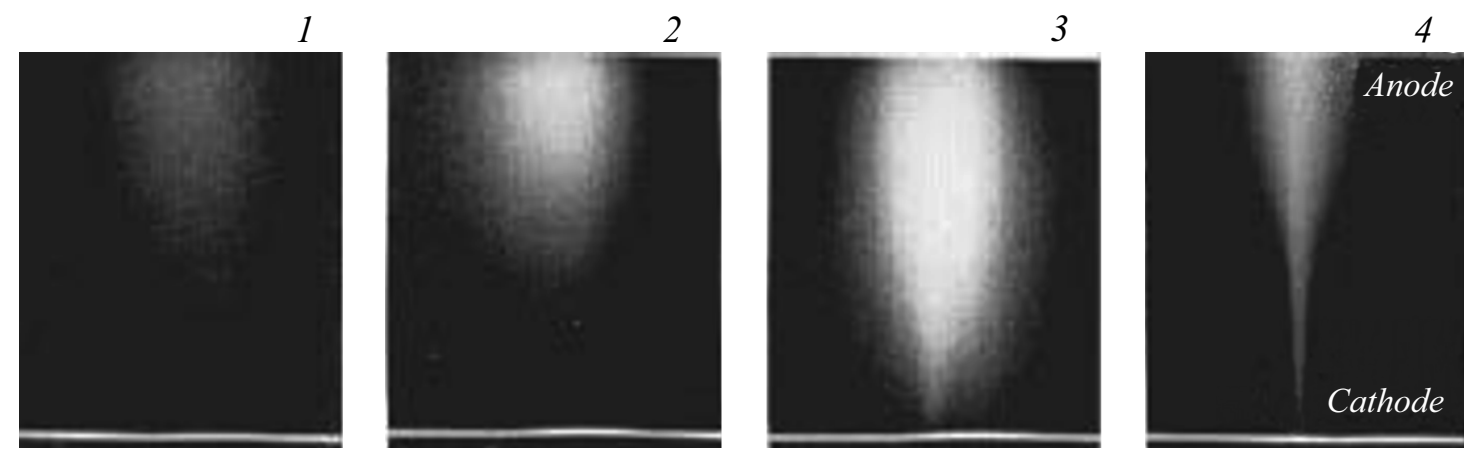

Рис. 1. Оптические картины развития разряда в аргоне: $p=760$ Torr, $U_{0}=6.8 \mathrm{kV}$. Цифры $1-4-$ номера кадров.

где $n, \Gamma, \mu, D-$ концентрация, поток, подвижность и коэффициент диффузии соответствующих компонент плазмы, $e-$ заряд электрона, $k_{\mathrm{B}}$ - постоянная Больцмана, $T_{e}, \lambda_{e}, D_{e}-$ температура, теплопроводность и коэффициент диффузии электронов, $n_{e}, n_{\mathrm{Ar}^{+}}, n_{\mathrm{Ar}_{2}^{+}}-$ концентрации электронов, атомарных и молекулярных ионов, $Q_{E}$ - работа электрического поля, $Q_{e l}, Q_{i n}-$ упругие и неупругие потери энергии электронов, $S-$ источник рождения и гибели рассматриваемых частиц в плазме, $\mathbf{E}$ - напряженность электрического поля. Для ионов $q=+1$, для электронов $q=-1$, для возбужденных частиц $q=0$.

Для диффузионного члена как в уравнении переноca, так и в уравнении энергии вместо общепринятых диффузионных потоков $\boldsymbol{\Gamma}_{\text {dif }}=-D \nabla n$ и $\mathbf{F}=-\lambda_{e} \nabla T_{e}$ используются правильные выражения: $\boldsymbol{\Gamma}_{d i f}=-\nabla(D n)$ и $\mathbf{F}=-\nabla\left(\lambda_{e} T_{e}\right)[11]$. Коэффициенты подвижности для ионов и коэффициент диффузии возбужденных частиц в собственном газе взяты из работы [12].

Граничные условия на катоде для потенциала, концентраций заряженных (индекс $i$ относится к атомарным и молекулярным ионам) и возбужденных $\left(n^{*}\right)$ частиц:

$$
\begin{gathered}
\varphi_{c}=0, \quad \frac{\partial n_{i}}{\partial z}=0, \boldsymbol{\Gamma}_{e}=-\gamma \sum_{i} \boldsymbol{\Gamma}_{i} \\
n^{*}=0, \frac{3}{2} k_{\mathrm{B}} T_{e}=I-2 \varphi_{W}
\end{gathered}
$$

на аноде:

$$
\varphi_{a}=0, \quad \frac{\partial n_{e}}{\partial z}=\frac{\partial T_{e}}{\partial z}=0, \quad n_{i}=0, \quad n^{*}=0 ;
$$

на боковых гранях расчетной области:

$$
\frac{\partial \varphi}{\partial r}=\frac{\partial n_{e}}{\partial r}=\frac{\partial n_{i}}{\partial r}=\frac{\partial n^{*}}{\partial r}=\frac{\partial T_{e}}{\partial r}=0,
$$

где $\gamma=0.1-$ второй коэффициент Таунсенда, $I=15.76 \mathrm{eV}-$ потенциал ионизации аргона, $\varphi_{W}=4.5 \mathrm{eV}$ - работа выхода катода. Для ионэлектронной эмиссии учитывался поток на катод как атомарных, так и молекулярных ионов.
Интегрирование проводилось явным методом [13] со вторым порядком точности по времени и пространству с числом Куранта 0.1. Уравнение Пуассона решалось итерационным методом переменных направлений.

Из анализа результатов моделирования следует, что электроны вследствие дрейфа уходят из прикатодной области в сторону анода, тем самым увеличивая концентрацию электронов в положительном столбе за счет ионизационного размножения (рис. 2,a,b). При этом образуется нескомпенсированный положительный объемный заряд (рис. 3,a), приводящий к усилению электрического поля $\mathbf{E}$ в прикатодной области с одновременным ослаблением $\mathbf{E}$ в положительном столбе (рис. $3, b$ ). По мере приближения к катоду напряженность поля на фронте ионизации также увеличивается, соответственно растет интенсивность ионизационных процессов.

Таким образом, формирование разряда в аргоне происходит за счет возникновения катодонаправленной волны ионизации. Оценим скорость движения фронта к катоду. Для этого рассмотрим положение двух минимумов электрического поля $E$ в разные моменты времени: $t_{1}=37 \mathrm{~ns}$ и $t_{2}=40 \mathrm{~ns}$ (рис. $3, b$ ). В результате получим скорость движения фронта на уровне $\sim 2.5 \cdot 10^{7} \mathrm{~cm} / \mathrm{s}$, что удовлетворительно согласуется с экспериментом.

На основании анализа пространственно-временно́го распределения концентрации возбужденных частиц $\mathrm{Ar}^{*}$ (рис. 2) можно сделать вывод, что в исследуемом диапазоне времен концентрация $\mathrm{Ar}^{*}$ непрерывно растет. Данное обстоятельство связано с тем, что источники рождения $\mathrm{Ar}^{*}$ в уравнении непрерывности (1) главным образом за счет возбуждения нейтральных атомов электронным ударом больше источника, отвечающего за гибель $\mathrm{Ar}^{*}$, куда входят деактивация возбужденных частиц при столкновении с электроном и нейтральной частицей, а также ступенчатая и пеннинговская ионизации.

В положительном столбе (рис. 2) концентрация молекулярных ионов $\mathrm{Ar}_{2}^{+}$хотя и меньше, чем у атомарных ионов $\mathrm{Ar}^{+}$, но также монотонно растет, что в свою очередь объясняется рождением $\mathrm{Ar}_{2}^{+}$в тройных столкновениях иона $\mathrm{Ar}^{+}$с нейтральными частицами, а разрушение несущественно, пока концентрация электронов мала. 
$a$
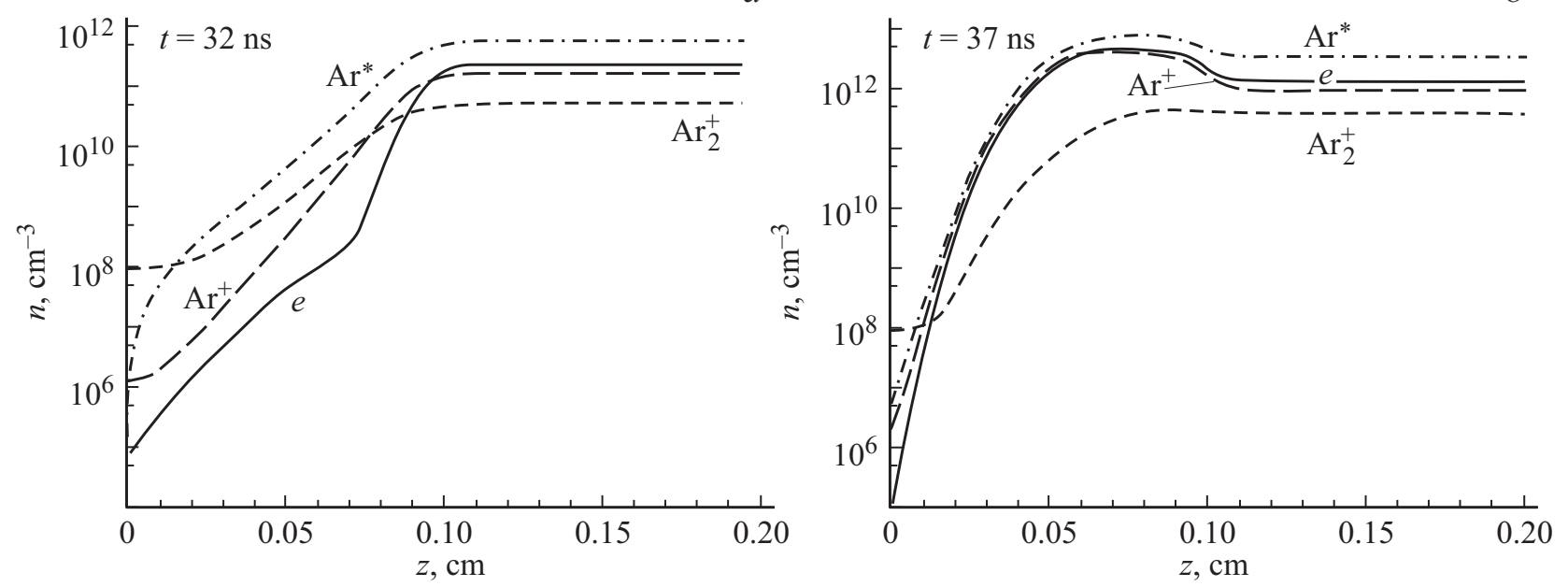

Рис. 2. Характерные распределения концентраций электронов $(e)$, ионов $\left(\mathrm{Ar}^{+}, \mathrm{Ar}_{2}^{+}\right)$и возбужденных атомов $\left(\mathrm{Ar}^{*}\right)$ аргона в межэлектродном промежутке для моментов времени $32(a)$ и $37 \mathrm{~ns}(b): p=760$ Torr, $U_{0}=6.8 \mathrm{kV}$.
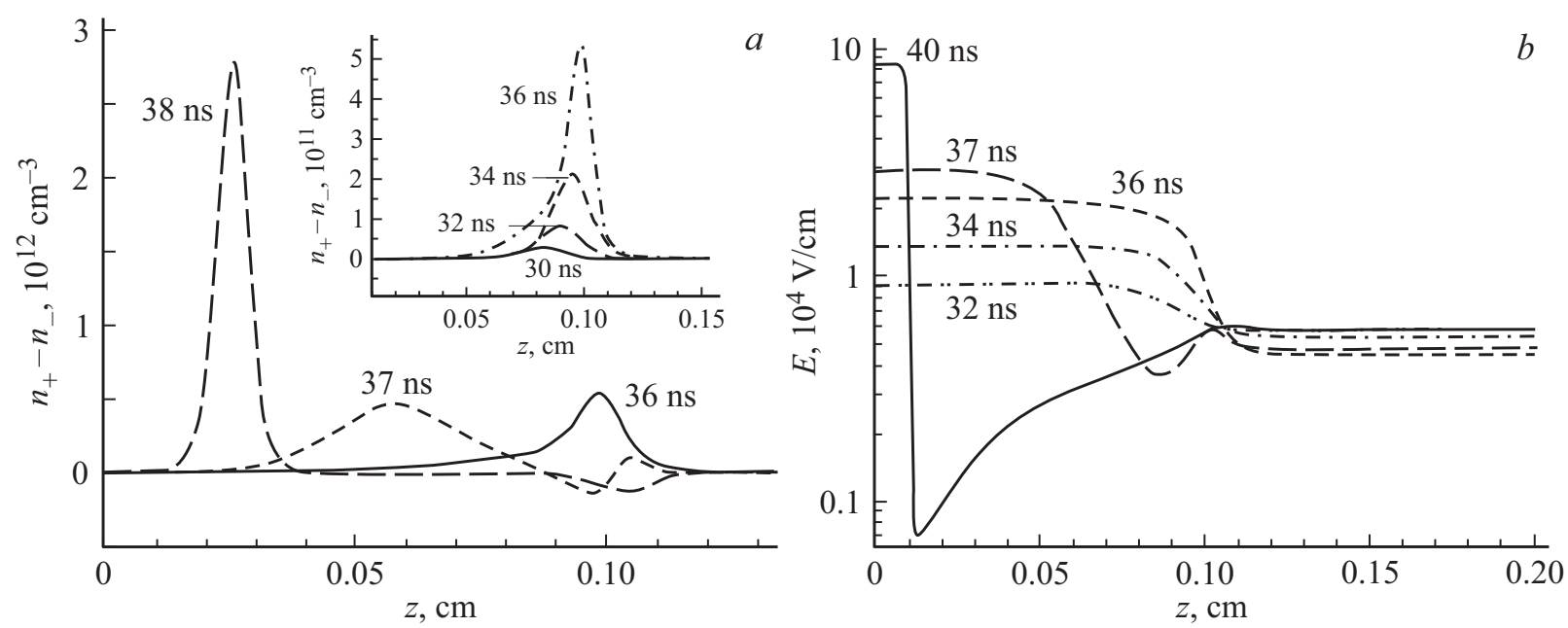

Pис. 3. Нескомпенсированный положительный пространственный заряд в межэлектродном промежутке в различные моменты времени $(a)$ и характерные распределения в межэлектродном промежутке напряженности электрического поля $(b): p=760$ Torr, $U_{0}=6.8 \mathrm{kV}$.

Начиная с $35 \mathrm{~ns}$ после приложения напряжения вблизи катода концентрации заряженных и возбужденных частиц, а также напряженность электрического поля ведут себя немонотонно (рис. $2, b$ и $3, b$ ). Кроме того, в прикатодной области существует подобласть, в которой концентрация возбужденных атомов оказывается ниже, чем концентрация молекулярных ионов (рис. 2,a), в то время как во всей расчетной области ситуация противоположная. Данное обстоятельство связано с постановкой граничных условий на катоде: концентрация $\mathrm{Ar}^{*}$ обращается в нуль. Изменение граничных условий для $\mathrm{Ar}^{*}$ может не только количественно, но и качественно изменить поведение профиля концентрации частиц, что в свою очередь может внести коррективы в формирование катодного слоя. При этом на больших временах нагрев газа окажется существенным и приведет к разрушению молекулярных ионов. Поэтому вопрос учета нагрева газа и отбора плазмохимических реакций требует дальнейших исследований.

Таким образом, в работе в результате моделирования показано, что формирование разряда начинается с катодонаправленной волны ионизации, которая движется со скоростью $\sim 2.5 \cdot 10^{7} \mathrm{~cm} / \mathrm{s}$, при этом к моменту времени $40 \mathrm{~ns}$ концентрация электронов в разрядном промежутке, за исключением катодного слоя, лежит в диапазоне $10^{13}-10^{14} \mathrm{~cm}^{-3}$, что удовлетворительно согласуется с результатами эксперимента, а напряженность поля близка к значению $10^{5} \mathrm{~V} / \mathrm{cm}$.

Работа выполнена при финансовой поддержке стипендии Президента РФ молодым ученым и аспирантам № СП-3812.2016.1 и гранта РФФИ № 18-08-00075a. 


\section{Список литературы}

[1] Королев Ю.Д., Месяи, Г.А. Физика импульсного пробоя газов. М.: Наука, 1991. $224 \mathrm{c}$.

[2] Тарасенко В.Ф., Бакшт Е.Х., Бураченко А.Г., Ломаев М.И., Сорокин Д.А., Шутько Ю.В. // ЖТФ. 2010. Т. 36. B. 8. C. $60-67$.

[3] Naidis G.V., Tarasenko V.F., Babaeva N.Yu., Lomaev M.I. // Plasma Sources Sci. Technol. 2018. V. 27. N 1. P. 013001.

[4] Осипов В.В. // УФН. 2000. Т. 170. № 3. С. 225-245.

[5] Курбанисмаилов В.С., Омаров О.А., Рагимханов Г.Б., Абакарова Х.М., Али А.Р.А. // Физика плазмы. 2016. Т. 42. № 7. C. $680-692$.

[6] Baeva M., Bosel A., Ehlbeck J. // Phys. Rev. E. 2012. V. 85. N 5. P. 056404.

[7] Терешонок Д.В. // Письма в ЖТФ. 2014. Т. 40. В. 3. С. $83-$ 89.

[8] Суржсков С.T. Физическая механика газовых разрядов. М.: Изд-во МГТУ им. Н.Э. Баумана, 2006. 640 с.

[9] Soloviev V.R., Krivtsov V.M. // J. Phys. D: Appl. Phys. 2009. V. 42. N 12. P. 125208.

[10] Курбанисмаилов В.С., Омаров О.А., Рагимханов Г.Б., Терешонок Д.В. // Письма в ЖТФ. 2017. Т. 43. В. 18. С. $73-$ 81.

[11] Hagelaar G.J.M., Pitchford L.C. // Plasma Sources Sci. Technol. 2005. V. 14. N 4. P. 722-733.

[12] Смирнов Б.М. Свойства газоразрядной плазмы. СПб.: Издво Политехн. ун-та, 2010. 361 с.

[13] Юргеленас Ю.В. // Журн. вычисл. математики и мат. физики. 2010. Т. 50. № 8. С. 1420-1437. 